\title{
Some Results on Stability of Difference Systems
}

\author{
XIAO-SONG YANG* and PING ZHOU \\ Institute for Nonlinear Systems, Chongqing University of Posts and Telecomm, Chongqing 400065, People's Republic of China
}

(Received 5 June 2001)

\begin{abstract}
This paper presents some new results on existence and stability of equilibrium or periodic points for difference systems. First sufficient conditions of existence of asymptotically stable equilibrium point as well as the asymptotic stability of given equilibrium point are given for second order or delay difference systems. Then some similar results on existence of asymptotically stable periodic (equilibrium) points to general difference systems are presented.
\end{abstract}

Keywords: Stability of equilibrium point; Difference systems; Second order systems; Dynamical system

\section{INTRODUCTION}

Difference dynamical systems are increasingly encountered in both natural and social sciences and have received much attention in recent years (Kocic and Ladas, 1993). A currently active area of research is the study of delay difference dynamical systems. For example, the second order (or delay) difference dynamical systems of the form

$$
y_{n+1}=f\left(y_{n}, y_{n-1}\right)
$$

has received lot of investigations in recent years (Fisher and Goh, 1984; Fisher, 1984; Sedaghat). Systems of type (Eq. (1.1)) often arose in some mathematical models of the macroeconomic systems and biological systems.

In this paper we deal with the existence of stable equilibrium points of nonlinear difference systems. For simplicity, we mainly focus on the second order nonlinear difference dynamical systems as well as the stability of given equilibrium points of the second order nonlinear difference systems. Also we discuss some general properties of two dimensional difference systems. The results obtained in this paper can be easily generalized to higher dimensional systems.

Due to the fact that the system shown in Eq. (1.1) can be transformed into a two dimensional difference system, we first review some preliminaries for two dimensional systems.

Consider the following difference system

$$
x_{n+1}=F\left(x_{n}\right), \quad x \in R^{2}, \quad x_{0}=x, \quad x \in R^{2},
$$

where $F$ is two dimensional continuous map, and $x$ is the initial condition.

DEFINITION 1.1 A point $\bar{x}$ is called an equilibrium point of Eq. (1.2), provided that it satisfies

$$
\bar{x}=F(\bar{x}) .
$$

A point $\bar{x}$ is called an periodic point of Eq. (1.2), if there exists a positive number $m$ such that

$$
F^{i}(\bar{x}) \neq \bar{x}, \quad i=1, \ldots, m-1
$$

and

$$
F^{m}(\bar{x})=\bar{x}
$$

DEFINITION 1.2 An orbit $\left\{x_{n}\right\}(n=0,1, \ldots$,$) with the$ initial condition $x_{0}=x$ is said to be stable, if it satisfies the following conditions:

For every $\varepsilon>0$, there exists $\delta>0$ such that if $\mid x-$ $y \mid<\delta$, then $\left|x_{n}-y_{n}\right|<\varepsilon$ for every orbit $\left\{y_{n}\right\}$ with the initial condition $y$.

DEFINITION 1.3 An orbit $\left\{x_{n}\right\}(n=0,1, \ldots)$ with the initial condition $x_{0}=x$ is said to be asymptotically stable, if it is stable and the following conditions hold:

$$
\left|x_{n}-y_{n}\right| \rightarrow 0, \text { as } n \rightarrow \infty \text {. }
$$

Definition 1.4 An orbit $\left\{x_{n}\right\}(n=0,1, \ldots$,$) with the$ initial condition $x_{0}=x$ is said to be uniformly

*Corresponding author. E-mail: yangxs@cqupt.edu.cn 
asymptotically stable, if it is stable and the following condition holds:

(1) For each $\varepsilon>0$ and integer $n>0$, there exists $\delta>0$ such that if $\left|x_{n}-y\right|<\delta$, then

$$
\left|x_{m+n}-y_{m}\right|=\left|F^{m}\left(x_{n}\right)-F^{m}(y)\right|<\varepsilon
$$

for every orbit $\left\{y_{n}\right\}$ with the above initial condition $y$. Furthermore, the following holds

(2) $\left|x_{m+n}-y_{m}\right|=\left|F^{m}\left(x_{n}\right)-F^{m}(y)\right| \rightarrow 0$, as $m \rightarrow \infty$.

\section{STABILITY OF THE SECOND ORDER DIFFERENCE EQUATION}

THEOREM 2.1 Suppose that the function $f(x, y)$ satisfies the following condition

$$
|f(\bar{x}, \bar{y})-f(x, y)| \leq a \max \{|\bar{x}-x|,|\bar{y}-y|\},
$$

where $a<1$ is a positive constant. Then Eq. (1.1) possesses a asymptotically stable equilibrium point.

Proof Let $\left\{y_{n}\right\}$ be an orbit of Eq. (1.1) with initial conditions $y_{0}$ and $y_{1}$. First it is easy to see that

$$
\begin{aligned}
\left|y_{2 n+1}-y_{2 n}\right| & =\left|f\left(y_{2 n}, y_{2 n-1}\right)-f\left(y_{2 n-1}, y_{2 n-2}\right)\right| \\
& \leq a \max \left\{\left|y_{2 n}-y_{2 n-1}\right|,\left|y_{2 n-1}-y_{2 n-2}\right|\right\} .
\end{aligned}
$$

In case of $\max \left\{\left|y_{2 n}-y_{2 n-1}\right|,\left|y_{2 n-1}-y_{2 n-2}\right|\right\}=$ $\left|y_{2 n}-y_{2 n-1}\right|$, one has

$$
\begin{aligned}
\left|y_{2 n+1}-y_{2 n}\right| & \leq a\left|y_{2 n}-y_{2 n-1}\right| \\
& \leq a^{2} \max \left\{\left|y_{2 n-1}-y_{2 n-2}\right|, \mid y_{2 n-2}\right.
\end{aligned}
$$

In case of $\max \left\{\left\{\left|z_{2 n}-3\right|\right\} y_{2 n-1}|,| y_{2 n-1}-y_{2 n-2} \mid\right\}=$ $\left|y_{2 n-1}-y_{2 n-2}\right|$, one has

$$
\begin{aligned}
\left|y_{2 n+1}-y_{2 n}\right| \leq & a\left|y_{2 n}-y_{2 n-1}\right| \\
\leq & a^{2} \max \left\{\left|y_{2 n-2}-y_{2 n-3}\right|, \mid y_{2 n-3}\right. \\
& \left.-y_{2 n-4} \mid\right\} .
\end{aligned}
$$

From these expressions it follows that

$$
\left|y_{2 n+1}-y_{2 n}\right| \leq a^{n} \max \left\{\left|y_{2}-y_{1}\right|,\left|y_{1}-y_{0}\right|\right\}
$$

Similarly one can show that

$$
\begin{aligned}
\left|y_{2 n+2}-y_{2 n+1}\right| & =\left|f\left(y_{2 n+1}, y_{2 n}\right)-f\left(y_{2 n}, y_{2 n-1}\right)\right| \\
& \leq a \max \left\{\left|y_{2 n+1},-y_{2 n}\right|, \mid y_{2 n}, y_{2 n-1}\right\} \\
& \leq a^{n} \max \left\{\left|y_{2}-y_{1}\right|,\left|y_{1}-y_{0}\right|\right\} .
\end{aligned}
$$

It is easy to see that $\left\{y_{n}\right\}$ is a Cauchy sequence, accordingly converging to finite point $\hat{y}$ which is clearly an equilibrium point of Eq. (1.1).

Now in view of Eq. (2.1) and the above arguments it is easy to show that every orbit $\left\{\bar{y}_{n}\right\}$ satisfies

$$
\left|\bar{y}_{2 n+1}-\hat{y}\right| \leq a^{n} \max \left\{\left|\bar{y}_{2}-\hat{y}\right|,\left|\bar{y}_{1}-\hat{y}\right|\right\},
$$

and

$$
\left|\bar{y}_{2 n+2}-\hat{y}\right| \leq a^{n} \max \left\{\left|\bar{y}_{2}-\hat{y}\right|,\left|\bar{y}_{1}-\hat{y}\right|\right\} .
$$

Therefore $\hat{y}$ is an (globally) asymptotically stable point of system (1.1).

As the consequence of the above theorem one has the following result.

COROLlary 2.2 If $f(x, y)$ satisfies

$$
|f(\bar{x}, \bar{y})-f(x, y)| \leq a|\bar{x}-x|+b|\bar{y}-y|,
$$

where $a>0, b>0$, and $a+b<1$. Then Eq. (1.1) possesses an asymptotically stable equilibrium point.

The following result is a consequence of linear analysis.

THEOREM 2.3 Let $\bar{y}$ be an equilibrium point of system (Eq. (1.1)). Suppose that at the point $\bar{y}$ the function $f(x, y)$ satisfies the following conditions:
(1) $\left|\frac{\partial f}{\partial x}\right| \leq 2\left|\frac{\partial f}{\partial y}\right|^{1 / 2}$,
(2) $\frac{\partial f}{\partial y}<0$,
(3) $\left|\frac{\partial f}{\partial y}\right|<1$.

Then $\bar{y}$ is asymptotically stable.

Proof Rewrite system (Eq. (1.1)) as

$$
x_{n+1}=f\left(x_{n}, y_{n}\right) \quad y_{n+1}=x_{n}
$$

Let $F(x, y)$ denote the map

$$
F(x, y)=\left(\begin{array}{c}
f(x, y) \\
x
\end{array}\right)
$$

then one has

$$
|J F-\lambda I|=\left|\begin{array}{cc}
\frac{\partial f}{\partial x}-\lambda & \frac{\partial f}{\partial y} \\
1 & -\lambda
\end{array}\right|=\lambda^{2}-\frac{\partial f}{\partial x} \lambda-\frac{\partial f}{\partial y},
$$

where $J F$ is the Jacobian matrix of $F(x, y)$. The corresponding eigenvalues are

$$
\lambda_{1,2}=\frac{\frac{\partial f}{\partial x} \pm \sqrt{\left(\frac{\partial f}{\partial x}\right)^{2}+4 \frac{\partial f}{\partial y}}}{2} .
$$

Because of $|(\partial f / \partial x)| \leq 2|(\partial f / \partial y)|^{1 / 2}$ and $(\partial f / \partial y)<0$ at the equilibrium point $\bar{y}$, it is easy to see that

$$
\lambda_{1,2}=\frac{\frac{\partial f}{\partial x} \pm \mathrm{i} \sqrt{-\left(\frac{\partial f}{\partial x}\right)^{2}-4 \frac{\partial f}{\partial y}}}{2},
$$


and at the point $\bar{y}$,

$$
\left|\lambda_{1,2}\right|^{2}=\frac{1}{4}\left[\left(\frac{\partial f}{\partial x}\right)^{2}-4 \frac{\partial f}{\partial y}-\left(\frac{\partial f}{\partial x}\right)^{2}\right]=-\frac{\partial f}{\partial y}<1 .
$$

This shows the $\bar{y}$ is (locally) asymptotically stable.

\section{FURTHER RESULTS ON DIFFERENCE SYSTEMS}

Consider a two-dimensional difference system

$$
x_{n+1}=F\left(x_{n}\right), \quad x \in R^{2} \quad x_{0} \in R^{2},
$$

where $F$ is a $C^{r}(r>0)$ function.

One can obtain the following result without difficulty.

THEOREM 3.1 Let A be a connected compact invariant set of Eq. (3.1). If there exists a constant $0<\delta<1$, such that

$$
|F(x)-F(\bar{x})| \leq \delta|x-\bar{x}|
$$

for every pair points $x$ and $\bar{x}$ of $A$, then $A$ is simply connected.

THEOREM 3.2 Let A be a compact invariant set of Eq. (3.1). Suppose that an orbit $\left\{x_{n}\right\} \subset A(n=0,1, \ldots$,$) of$ Eq. (3.1) is uniformly asymptotically stable, then Eq. (3.1) has an asymptotically stable periodic orbit.

Proof Because of the compactness of the invariant set $A$, there exists a sequence $n_{i} \rightarrow \infty$ such that

$$
\lim _{t \rightarrow \infty} x_{n_{t}}=\bar{x}
$$

Therefore, for the $\delta>0$ in Definition 1.4, there exists a $J>0$, such that in case of $j>J$, one has

$$
\left|x_{n_{j+1}}-x_{n_{j}}\right|<\delta
$$

Because of the uniform asymptotic stability of the orbit $\left\{x_{n}\right\}$, it is easy to see that

$$
\left|x_{m+n_{j+1}}-x_{m+n_{j}}\right| \rightarrow 0, \quad \text { as } m \rightarrow \infty
$$

Particularly, one has for the sequence $n_{i}$ that

$$
\left|x_{n_{i}+n_{j+1}}-x_{n_{i}+n_{j}}\right| \rightarrow 0, \quad \text { as } i \rightarrow \infty .
$$

This implies that

$$
\left|F^{n_{j+1}}\left(x_{n_{i}}\right)-F^{n_{j}}\left(x_{n_{i}}\right)\right| \rightarrow 0, \quad \text { as } i \rightarrow \infty .
$$

i.e.

$$
\left|F^{n_{j+1}}(\bar{x})-F^{n_{j}}(\bar{x})\right|=0,
$$

showing that $\bar{x}$ is a periodic orbit of period of $n_{j+1}-n_{j}$. It is apparent that $\bar{x}$ is asymptotically stable.
COROLlaRY 3.3 Suppose that for a nontrivial orbit $\left\{x_{n}\right\}$ of Eq. (3.1), the condition that there exists a constant $0<\delta<1$, such that $\left|F\left(x_{n+1}\right)-F\left(x_{n}\right)\right| \leq \delta\left|x_{n+1}-x_{n}\right|$ is satisfied, then Eq. (3.1) has an asymptotically stable period (equilibrium) orbit.

THEOREM 3.4 Let $A$ be a compact subset of $R^{2}$ which is invariant under $F$ in Eq. (3.1). Suppose that $F$ is continuously differentiable on $A$ and every eigenvalue $\lambda(x)$ of JF satisfies

$$
|\lambda(x)|<1, \quad x \in A,
$$

where JF is the Jacobian matrix of F. Then system (Eq. (3.1)) has an asymptotically stable (periodic) equilibrium point in A.

Proof First it is easy to see that $\lambda(x)$ is continuous on $A$ by virtue of the conditions given. Now because of inequality (Eq. (3.3)), and by means of theory of linear systems, there exists a positive continuous function $\eta(x)<1$ and a metric function (Riemannian metric) $K_{x}($,) with

$$
K_{x}(V, V)=\left(V^{t} Q(x) V\right), \quad x \in A .
$$

where $Q(x)$ is a positive symmetric matrix (continuous) function of $x$, such that for any vector $V \in R^{2}$,

$$
K_{F(x)}(J F(x) V) \leq \eta^{2} K_{x}(V, V)
$$

From the compactness of $A$, it is easy to see that there is positive number $\delta<1$ such that $\eta(x) \leq \delta<1$.

It follows that every orbit $\left\{x_{n}\right\} \subset \mathrm{A}(n=0,1, \ldots$,$) of$ Eq. (3.1) is uniformly asymptotically stable. Therefore, in the same manner as in the proof of the Theorem 3.2, we get the conclusion that system (Eq. (3.1)) has an asymptotically stable periodic (equilibrium) point in A.

Corollary 3.5 Let $A$ be an invariant compact set of system (Eq. (2.2)). Suppose that at every point $(x, y) \in A$ the function $f(x, y)$ satisfies the following conditions:

$$
\text { (1) }\left|\frac{\partial f}{\partial x}\right| \leq 2\left|\frac{\partial f}{\partial y}\right|^{1 / 2}, \quad \text { (2) } \frac{\partial f}{\partial y}<0, \quad \text { (3) }\left|\frac{\partial f}{\partial y}\right|<1 .
$$

Then system (Eq. (2.2)) has an asymptotically stable periodic (equilibrium) point.

\section{References}

Fisher, M.E. (1984) "Stability of a class of delay difference equation", Nonlinear Anal. 8, 645 .

Fisher, M.E. and Goh, B.S. (1984) "Stability results for delayed recritment in population dynamics", J. Math. Biol. 19, 147.

Kocic, V.L. and Ladas, G. (1993) Global Behavior of Nonlinear Difference Equation of Higher Order With Applications (Kluwer Academic Publishers, Boston).

Sedahat, H. (1997) "A class of nonlinear second order difference equations from macroeconomics", Nonlinear Analysis, Theory, Methods and Applications 5 (29), 593-603. 


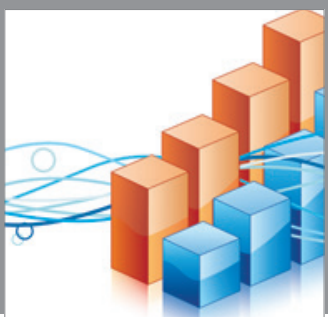

Advances in

Operations Research

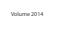

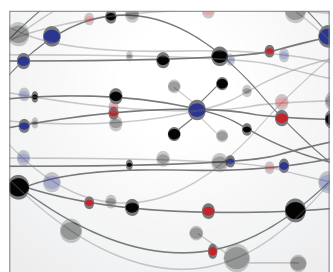

\section{The Scientific} World Journal
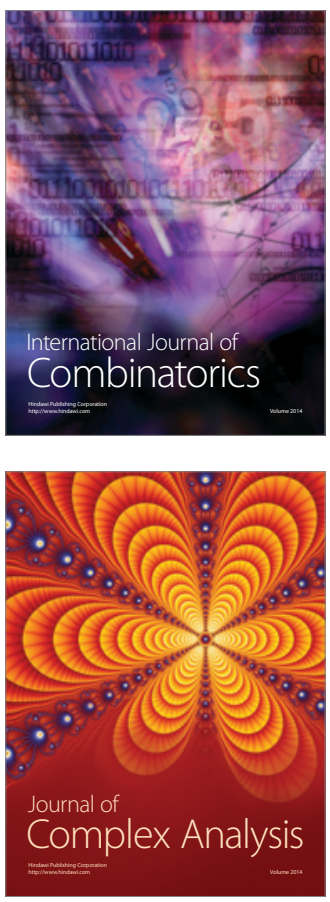

International Journal of

Mathematics and

Mathematical

Sciences
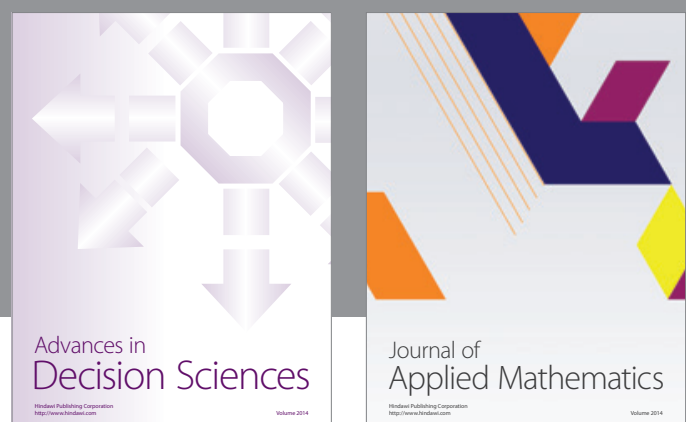

Journal of

Applied Mathematics
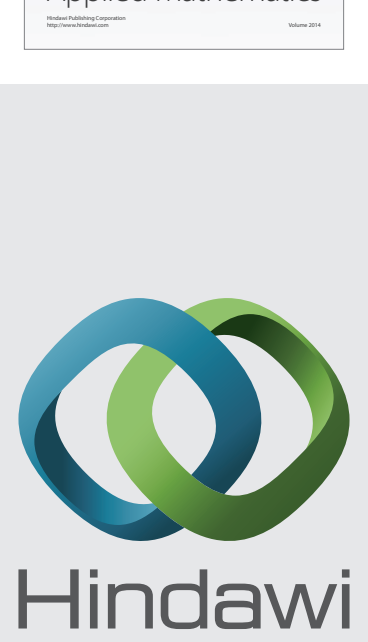

Submit your manuscripts at http://www.hindawi.com
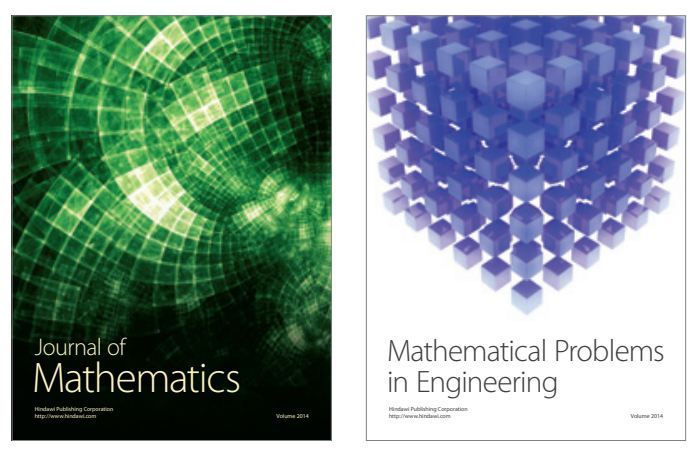

Mathematical Problems in Engineering
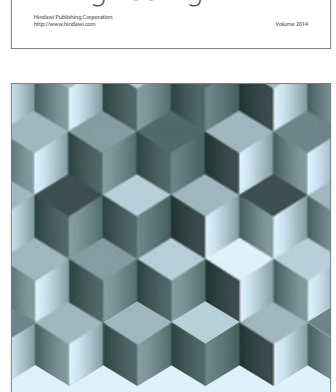

Journal of

Function Spaces
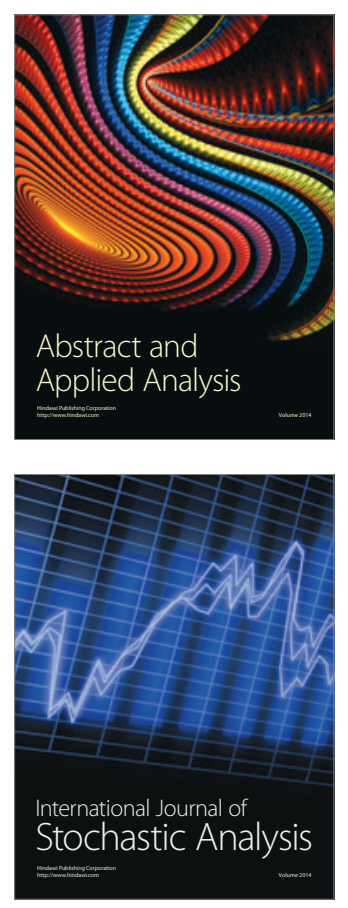

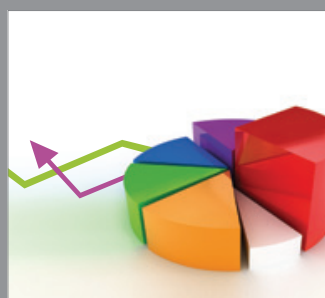

ournal of

Probability and Statistics

Promensencen
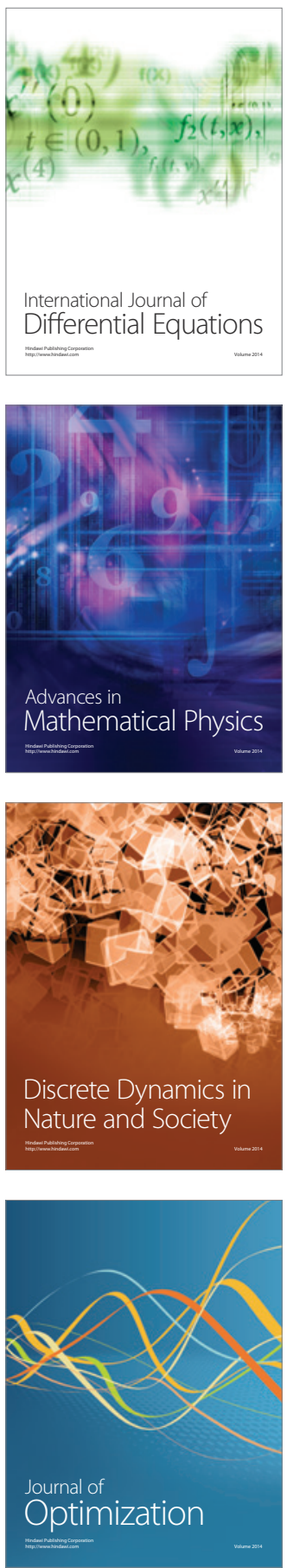